\title{
Nutrient intake and dietary pattern among pregnant women visiting the tertiary hospital of Nepal
}

Amit Timilsina $^{1^{*}}$, Anisha Shrestha ${ }^{2}$, Rajan Paudel ${ }^{2}$

${ }^{1}$ Master of Science in Public Health (MscPH), University of Southern Denmark, Esbjerg, Denmark.

${ }^{2}$ Bachelor in Public Health (BPH), Institute of Medicine, Tribhuvan University, Kathmandu, Nepal.

Received:

22 February 2020

Revised:

1 November 2020

Accepted:

2 November 2020

\begin{abstract}
Background: Pregnancy demands adequate nutritional requirements for foetal growth, good health of pregnant women and to avoid adverse effects during pregnancy. Women from developing countries with limited resources have inadequate consumption of nutritional diets. This paper assesses the nutrient intake and dietary pattern of Nepalese pregnant women compared with recommended nutrient intake. This paper also observes trimester wise nutrient intake among pregnant women in Nepal.
\end{abstract}

Methodology: A descriptive cross-sectional study was conducted among 323 pregnant women who visited Gynaecology and Obstetrics Out-Patient Department (OPD) at Tribhuvan University Teaching Hospital (TUTH) for regular Antenatal Care (ANC) check-ups. Systematic random sampling methods were followed and data were collected from September 2016 to October 2016. Nutrient intake was evaluated with a 24-hour dietary recall method and compared with the Nepalese Food Composition Table 2017 for available nutrients. Face-to-face structured interviews were conducted to assess the nutrient intake of the respondents. The data were entered in Microsoft Excel 2007 and statistical analysis was carried out in SPSS v26. The descriptive analysis was done to identify the distribution of socio-demographic variables of pregnant women. The one-way Analysis of Variance (ANOVA) was used to compare the mean of nutrient intake distribution while Tukey's post-hoc test was done to compare trimester-wise nutrient intakes.

Results: Except for fat, the intake of nutrients were below the Nepalese Recommended Nutrient Intake. Carbohydrate and energy intake in the second and third trimester was significantly different from that in the first trimester $(\mathrm{p}=0.006$ and 0.004 respectively for carbohydrates and $\mathrm{p}=0.009$ and 0.002 respectively for energy intake). However, riboflavin intake was significantly lower only in the third trimester as compared to the first ( $\mathrm{p}=0.025$ ) while there were no significant intake differences between the first and second trimester and second and third trimester. No significant trimester wise differences were observed for intake distribution of other nutrients. Cereal intake was found to be abundant in the diet of pregnant women followed by pulses, legumes, and other vegetables.

Conclusion: The research concludes inadequate nutrient intake among pregnant women as compared to the national Recommended Nutrient Intake.

Keywords: dietary pattern, nutrient intake, pregnant women, Nepal

Tweetable Abstract: Nutrient intake for fat only met recommended nutrient intake among pregnant women receiving Antenatal Care service of Nepal.

\section{Introduction}

The amount of each nutrient needed for the physiological process such as pregnancy depends upon a person's physical factors such as age, weight and psychological factors during pregnancy demands for high nutrition [1]. Pregnancy demands for the adequate requirement of nutrients than any other life course for positive maternal outcome such as healthy foetal growth, expansion of maternal tissue and to decrease the likelihood of unfavourable pregnancy outcomes such as premature birth and/or low birth weight $[2,3,4,5]$. Inadequate supplementation of nutrients during pregnancy has been one of the public health problems around the globe and South Asia including Nepal [6, 7].

Research on dietary patterns in poor resource settings shows abundant cereal consumption and plant-based nutrient intake but occasional consumption of a balanced diet during pregnancy $[7,8]$. A large number of pregnant and postpartum women face a number of food proscriptions or food taboos according to religion and culture that contributes to inadequate consumption of diet among women and children [6]. Anorexia nervosa and bulimia nervosa are serious eating disorders that may begin in pregnancy in a poor nutritional state [9]. Prolonged deficiency of vitamin D may increase the risk of pre-eclampsia, low birth weight, neonatal hypocalcaemia, poor postnatal growth, bone fragility, and an increased incidence of autoimmune diseases during and after pregnancy [10]. Balanced energy and protein dietary intake 
is essential for pregnant women to prevent the risk of stillbirth and small-for-gestational-age neonates [11]. During pregnancy, blood levels of water-soluble vitamins decline significantly and foetal blood levels become greater than those in maternal blood, reflecting active placental transport of the vitamins [12]. Anaemia caused due to a deficiency of iron can lead to an increased rate of maternal morbidity, mortality, and poor birth outcomes [13]. The previous study highlights inadequate consumption of nutritional diet during pregnancy $[7,14,15,16]$ and reports the association of low birth weight of children with insufficient nutrient intake such as riboflavin, folic acid and vitamin $\mathrm{C}$ during the third trimester [16]. The anaemia rate is higher for pregnant women and lactating mothers (46\%) than non-pregnant and non-lactating women (39\%) [13]. The Nepal Demographic Health Survey (NDHS) 2016 also reports $12 \%$ to have low birth weight (below $2.5 \mathrm{~kg}$ ) among children with reported birth weight [13].

There are very limited numbers of research conducted in Nepal on nutrient intake among pregnant women [17]. This research assesses nutrient intake among pregnant women visiting ANC service at TUTH comparing it with Recommended Nutrient Intake (RNI) as proposed by Nepalese Food Composition Table 2017. The RDI is the average daily nutrient intake level that is sufficient to meet the nutrient requirement of nearly all ( 97 to $98 \%$ ) healthy individuals in a particular gender and life stage group [18]. This paper seeks to assess the nutrient intake and dietary pattern among Nepalese pregnant women compared with RNI. This paper also observes that trimester-wise nutrients intake among pregnant women as trimester wise recommended intake of nutrient is related to positive maternal outcome [19].

\section{Methods}

\section{Study design and sample design}

The descriptive cross-sectional study was designed to assess nutrient intake among pregnant women who visited the Gynaecology and Obstetrics Out-Patient Department (OPD) at Tribhuvan University Teaching Hospital (TUTH) for regular Antenatal Care (ANC) check -ups. The expected pregnancy of Kathmandu district is higher than any other district in Nepal [20]. TUTH is one of the major central level and referral hospitals with high patient flow [21]. Information of nutrient and dietary intake was collected through an interviewer-administered questionnaire. Data collection was done after the development of protocol and detailed orientation among the researchers. Pregnant women of age group 18 and above of any trimester were recruited in this study with a systematic random sampling method. The sample size was determined to be 327 with the assumption that $48 \%$ [22] of pregnant women won't meet nutritional needs (dietary intake) during pregnancy, with a standard normal deviate $(\mathrm{z})$ set at 1.96 (95\% confidence level) with additional $10 \%$ non-response rate. The monthly data of pregnant women visiting TUTH for ANC service was taken from the hospital record and $\mathrm{k}^{\text {th }}$ terms were determined accordingly. Based on the waiting list for ANC check-up for the particular day, every $3^{\text {rd }}$ patient was interviewed.

\section{Data collection}

The 24-hour dietary recall (24hDR) method was adopted and implemented. The 24-hour dietary recall method is one of the most utilized methods in nutritional research which involves recalling, describing and quantifying the intake of foods and beverages consumed in the last 24 hours [15, 16, 23] For this, semi-structured 24-hour dietary questionnaire was adopted based on Gibson and Ferguson (2008) [24]. Questions related to socio-demographic status were added and the translated questionnaire was pretested among pregnant women who visited the afternoon shift of TUTH.

The data was collected in the month of September and October in 2016. Face-to-face structured interviews were conducted to assess nutrient intake of respondents. The participants were asked about the type of food items and beverages they consumed for the last 24 hours with the quantity (number of standardized servings each time) and frequency (times per day). To measure the amount of food and beverages intake by pregnant women; respondents were shown visual pictures of food proportions and measuring instruments such as cups, bowls, plates, and spoons were shown for estimation of food proportion.

In the case of fasting, the information on the previous day intake of food was taken. The study was conducted according to the guidelines in the Declaration of Helsinki [25]. Participants were asked for written consent before being interviewed for their approval to participate in this research study. Similarly, participants were explained about the process, objective, and were informed to skip the question or the whole interview if they felt uncomfortable. Ethical approval was taken from the Institutional Review Board (IRB), Maharajgunj Medical Campus and approval was also taken from the administrative department of TUTH.

\section{Data Processing and Analysis}

Nepal does not have a food guide of its own and thus adopts Indian Dietary Recommendation (IDR) as a reference to develop Nepalese Food Composition Table [26]. The nutrient intakes for this research were estimated and quantified using Nepalese Food Composition Table 2017 [26]. Only the values of nutrients for energy, protein, fat, calcium, iron, vitamin $\mathrm{C}$, thiamine, riboflavin, niacin were calculated as food composition only provides value for those nutrients. The values of nutrients whose data were not available for food commodities in Nepalese Food Composition table 2017 were not calculated for this research.

Data was entered into Microsoft Excel 2007 and analysed using the Statistical Package for Social Sciences (SPSS) v 26. Out of 327 participants, only 323 participants had given complete interviews. The descriptive analysis was done to identify the distribution of socio-demographic variables of pregnant women. Intake distributions were compared using a one-way Analysis of Variance (ANOVA) test at $5 \%$ level of significance, considering $p<0.05$ as statistically significant, while Tukey's post-hoc test was done for trimester wise comparisons. Overall energy and nutrition intakes were compared to the RNI values provided by the Nepalese Food Composition Table 2017, using one sample t-test at 5\% level of significance, considering $\mathrm{p}<0.05$ as statistically significant.

\section{Results}

\section{Demographic characteristics}

A total of 323 pregnant women in all trimesters were included in the analysis as illustrated in Table 1 . The distribution of the participants according to trimester shows that $11 \%$ of them were in the 
first trimester, 33\% were in the second trimester, and the remaining $66 \%$ were in their third trimester. The mean age of the participants was $25.35 \pm 4.07$ years with most of them following Hinduism $(85.8 \%)$. The average family size was $4.69 \pm 2.5$. The mean age at marriage was found to be $21.55 \pm 3.4$ and $28 \%$ of the respondents were below the age of 20 years. The average weight of the mother was $56.38 \pm 9.28 \mathrm{~kg}$. Only $1.9 \%$ of the respondents had never received any type of education. 209 of the participants were in their first pregnancy while 100 participants had at most 2 children and 14 of the respondents had more than 2 children.

Table 1: Demographic characteristics of study participants

\begin{tabular}{|c|c|c|}
\hline Characteristics & Number & Percentage \\
\hline \multicolumn{3}{|l|}{ Age } \\
\hline$<=19$ & 14 & 4.3 \\
\hline $20-24$ & 138 & 42.7 \\
\hline $25-29$ & 115 & 35.6 \\
\hline $30-34$ & 50 & 15.5 \\
\hline $35-39$ & 6 & 1.9 \\
\hline \multicolumn{3}{|l|}{ Trimester } \\
\hline 1 st & 36 & 11.2 \\
\hline 2nd & 107 & 33.1 \\
\hline 3 rd & 180 & 55.7 \\
\hline \multicolumn{3}{|l|}{ Age at Marriage } \\
\hline$<20$ & 89 & 28.0 \\
\hline$>=20$ & 229 & 72.0 \\
\hline \multicolumn{3}{|l|}{ Religion } \\
\hline Hindu & 277 & 85.8 \\
\hline Buddhist & 30 & 9.2 \\
\hline Christian & 10 & 3.2 \\
\hline Others & 6 & 1.8 \\
\hline \multicolumn{3}{|l|}{ Education Level } \\
\hline Uneducated & 6 & 1.9 \\
\hline Vocational Education & 3 & 0.9 \\
\hline Primary level & 10 & 3.1 \\
\hline Lower Secondary & 41 & 12.7 \\
\hline Secondary & 81 & 25.1 \\
\hline Higher Secondary & 96 & 29.7 \\
\hline Bachelor & 71 & 22.0 \\
\hline Master & 15 & 4.6 \\
\hline \multicolumn{3}{|l|}{ No. of children } \\
\hline No Child & 209 & 64.7 \\
\hline $1-2$ & 100 & 31.0 \\
\hline More than 2 & 14 & 4.3 \\
\hline
\end{tabular}

\section{Nutrient intake}

The overall nutrient intake distributions were compared with the Recommended Nutrient Intake using one sample ' $\mathrm{t}$ ' test as shown in Table 2. The overall protein intake was less $(81.2 \pm 28.3$ $\mathrm{gm})$ than the RNI (82.2 gm); it was not statistically significant (p $=0.557)$. The energy $(2331.9 \pm 588.5 \mathrm{kcal})$ intake was significantly lower $(\mathrm{p}<0.001)$ than the RNI for energy $(2580 \mathrm{kcal})$. The fat intake $(42.4 \pm 19.1 \mathrm{gm})$ was significantly higher $(\mathrm{p}<0.001)$ than the RNI fat (30 gm). On the contrary, calcium $(486.4 \pm 349.8 \mathrm{mg}$ compared to $1200 \mathrm{mg}$ ), iron $(21.7 \pm 46.6 \mathrm{mg}$ compared to $35 \mathrm{mg})$, vitamin $\mathrm{C}$ (19.1 $\pm 20.7 \mathrm{mg}$ compared to $60 \mathrm{mg})$, thiamine $(0.1 \pm 0.3 \mathrm{mg}$ compared to $1.3 \mathrm{mg})$, riboflavin $(0.3 \pm 0.9 \mathrm{mg}$ compared to $1.6 \mathrm{mg})$ and niacin $(1.7 \pm 2.3 \mathrm{mg}$ compared to $16 \mathrm{mg})$ intakes were found to be significantly less $(\mathrm{p}<0.001)$ than the RNI.

Table 2: Comparison of overall intake with recommended nutrient intake (per day) $(n=323)$

\begin{tabular}{lccc}
\hline Energy and nutrients & Overall & RNI & P value \\
\hline Protein & $81.2 \pm 28.3$ & $82.2 \mathrm{gm}$ & 0.557 \\
Fat & $42.4 \pm 19.1$ & $30 \mathrm{gm}$ & $<0.001^{*}$ \\
Energy & $2331.9 \pm 588.5$ & $2580 \mathrm{kcal}$ & $<0.001^{\star}$ \\
Calcium & $486.4 \pm 349.8$ & $1200 \mathrm{mg}$ & $<0.001^{*}$ \\
Iron & $21.7 \pm 46.6$ & $35 \mathrm{mg}$ & $<0.001^{\star}$ \\
Thiamin (Vit. B1) & $0.1 \pm 0.3$ & $1.3 \mathrm{mg}$ & $<0.001^{*}$ \\
Riboflavin (Vit. B2) & $0.3 \pm 0.9$ & $1.6 \mathrm{mg}$ & $<0.001^{\star}$ \\
Niacin (Vit. B3) & $1.7 \pm 2.3$ & $16 \mathrm{mg}$ & $<0.001^{\star}$ \\
Ascorbic Acid (Vit. C) & $19.1 \pm 20.7$ & $60 \mathrm{mg}$ & $<0.001^{*}$ \\
\hline
\end{tabular}

${ }^{\star}$ Statistically significant at $5 \%$ level of significance

The dietary pattern of pregnant women as shown in Table 3 shows 931.86 gm per person per day consumption of cereals, $405.01 \mathrm{gm}$ per person per day consumption of pulses and legumes, $201.80 \mathrm{gm}$ per person per day consumption of vegetables and 331 gm per person per day consumption of fruits on an average. Similarly, 253.56 gm per person per day consumption of milk, 147.98 gm per person per day consumption of meat and fish, 1.1 gm per person per day consumption of egg and $0.01 \mathrm{gm}$ per person per day consumption of green leafy vegetable was also observed.

Table 3: Per day gram intake of different food source $(\mathbf{n}=323)$

\begin{tabular}{lcc}
\hline Food Group & $\begin{array}{c}\text { Average Intake } \\
\text { (gm/day) }\end{array}$ & $\begin{array}{c}\text { Distribution of } \\
\text { food source (\%) }\end{array}$ \\
\hline Cereals & 931.87 & 39.3 \\
Pulses and Legumes & 405.01 & 17.1 \\
Green Leafy Vegetables & 201.80 & 8.5 \\
Other Vegetables & 331.77 & 14.0 \\
Fruits & 253.57 & 10.7 \\
Milk and Milk Products & 149.15 & 6.3 \\
Meat or Fish & 79.63 & 3.4 \\
Egg & 16.72 & 0.7 \\
Others (Roots, Tubers & 0.4 & 0.017 \\
and Nuts) & &
\end{tabular}

The intake distributions for energy and nutrients were illustrated as mean intake distributions as shown in Table 4. Carbohydrate 
intake in the second and third trimester was significantly different ( $p=0.006$ and 0.004 respectively) with that in the first trimester. Likewise, energy intake was also significantly higher in the second and third trimester as compared to the first trimester $(\mathrm{p}=0.009$ and 0.002 respectively). However, riboflavin intake was significantly lower $(\mathrm{p}=0.025)$ only in the third trimester as compared to first whereas no significant difference was found in intake in first and second trimesters. No significant trimester wise differences were observed for intake distribution of other nutrients. The energy was found to be derived from carbohydrates (71.26\%), fat (15.48\%) and protein (13.24\%) as shown in Table 4. reduce the risk of premature birth and is important for optimal visual and cognitive development [28]. However, comparatively less consumption of protein and energy has been associated with low pregnancy weight gain and impacts foetal growth and thus it is important for pregnant women to have adequate consumption of protein and energy containing food [27]. Even though abundant cereal consumption was observed, the dietary intake is still not enough to meet the energy intake of pregnant women. A major proportion of energy was derived from carbohydrates followed by fat and protein. However, it is to note that no comparison of carbohydrate intake was assessed due to unavailability of RNI for car-

Table 4: Energy and nutrient intake among pregnant women ( $24 \mathrm{hr}$. recall)

\begin{tabular}{lccccc}
\hline Energy and nutrients & $\begin{array}{c}\text { First Trimester } \\
(\mathbf{n}=\mathbf{3 6})\end{array}$ & $\begin{array}{c}\text { Second Trimester } \\
(\mathbf{n}=\mathbf{1 0 7})\end{array}$ & $\begin{array}{c}\text { Third Trimester } \\
(\mathbf{n}=\mathbf{1 8 0})\end{array}$ & $\begin{array}{c}\text { Overall } \\
(\mathbf{n}=\mathbf{3 2 3})\end{array}$ & P value \\
\hline Energy & $2021.8 \pm 597.7$ & $2354.9 \pm 592.9$ & $2380.3 \pm 568.4$ & $2331.9 \pm 588.5$ & $0.003^{*}$ \\
Protein & $72.6 \pm 31.5$ & $81.3 \pm 27.8$ & $82.9 \pm 27.7$ & $81.2 \pm 28.3$ & 0.138 \\
Fat & $37.9 \pm 19.8$ & $42 \pm 20.1$ & $43.5 \pm 18.3$ & $42.4 \pm 19.1$ & 0.265 \\
Carbohydrates & $379.8 \pm 117.7$ & $446.9 \pm 112.1$ & $445.7 \pm 110.4$ & $438.7 \pm 113.4$ & $0.004^{*}$ \\
\% of Energy from Protein & $13.5 \pm 3.9$ & $13.0 \pm 3.0$ & $13.2 \pm 3.3$ & $13.2 \pm 3.2$ & 0.789 \\
\% of Energy from Carbohydrates & $70.5 \pm 7.6$ & $71.9 \pm 6.4$ & $71.0 \pm 6.2$ & $71.3 \pm 6.5$ & 0.385 \\
\% of Energy from Fat & $15.9 \pm 6.6$ & $14.9 \pm 5.5$ & $15.7 \pm 5.6$ & $15.5 \pm 5.7$ & 0.500 \\
Minerals & $22.9 \pm 7.6$ & $24.5 \pm 8.3$ & $24.2 \pm 7.1$ & $24.2 \pm 7.6$ & 0.544 \\
Fiber & $176.4 \pm 59.3$ & $200 \pm 96.7$ & $197.7 \pm 84.5$ & $196.1 \pm 86.5$ & 0.345 \\
Calcium & $390.2 \pm 284.5$ & $491.4 \pm 353$ & $502.6 \pm 358.3$ & $486.4 \pm 349.8$ & 0.209 \\
Phosphorous & $504.3 \pm 340.8$ & $534.1 \pm 419.8$ & $564.1 \pm 372.8$ & $547.5 \pm 385.1$ & 0.634 \\
Iron & $11.1 \pm 11.1$ & $24.3 \pm 49.9$ & $22.3 \pm 48.8$ & $21.7 \pm 46.6$ & 0.332 \\
Carotene & $322.2 \pm 293.4$ & $499.2 \pm 595.2$ & $508.9 \pm 687.8$ & $484.9 \pm 626.4$ & 0.254 \\
Thiamine (Vit. B1) & $0.31 \pm 0.71$ & $0.2 \pm 0.3$ & $0.1 \pm 0.2$ & $0.1 \pm 0.3$ & 0.091 \\
Riboflavin (Vit. B2) & $0.7 \pm 2.6$ & $0.3 \pm 0.2$ & $0.28 \pm 0.22$ & $0.3 \pm 0.9$ & $0.030^{*}$ \\
Niacin (Vit. B3) & $2.2 \pm 3$ & $1.6 \pm 2.1$ & $1.6 \pm 2.3$ & $1.7 \pm 2.3$ & 0.311 \\
Ascorbic Acid (Vit. C) & $15.5 \pm 19.5$ & $21.4 \pm 21.9$ & $18.5 \pm 20.2$ & $19.1 \pm 20.7$ & 0.285 \\
\hline
\end{tabular}

*Statistically significant at $5 \%$ level of significance

\section{Discussion}

Pregnancy is a period of high demand nutrients for healthy weight gain, child birth weight and to reduce pregnancy related complications. Nepal adopts references regarding nutrient composition, and Recommended Nutrient Intake and allowances from IDR as stated in Nepalese Food Composition Table 2017 [26]. From a public health perspective, the nutrient intakes of pregnant women should meet the recommended nutrient intake to reduce infant mortality and maternal mortality rate. This study shows that only fat consumption has met the recommended nutrient intake. The consumption of protein and energy was found to be inadequate and these results are consistent with findings of previous studies conducted in Nepal and India $[15,27]$. This suggests an increased consumption of energy giving foods during pregnancy. The protein intake has been found to be high in this research as compared to previous research conducted in Nepal [27]. The consumption of milk and milk products and use of oil to prepare food is assumed to help to meet RNI for fat. Adequate fat consumption helps to bohydrates. Balanced protein-energy nutrients during pregnancy are very important for foetal growth and to reduce neonatal death, and thus protein-energy balance in the diet needs to be maintained [29]. Our study indicates low intake of iron among pregnant women, which might increase the risk of maternal anaemia, puerperal sepsis, low birth weight and preterm birth. This result is in line with the national data, as the report of the NDHS 2016 shows the high prevalence of iron deficiency anaemia among pregnant women. Thus, supplementation of iron during pregnancy should be encouraged $[10,13]$. The nutrient intake is also inadequate for calcium, vitamin C (ascorbic acid), vitamin B1 (thiamine), vitamin B2 (riboflavin) and vitamin B3 (niacin) consumption.

This study shows an imbalanced nutrient intake among pregnant women as the average nutrient intake has not met the recommended nutrient intake except for fat. Furthermore, the diet of pregnant women was not balanced as consumption of cereals, pulses and legumes are higher than green leafy vegetables, fruits, milk products, 
meat and fish. The result is consistent with previous research conducted in Nepal, however the milk and milk products were found to be consumed higher than the findings of this research [27]. Also, research conducted in India and systematic review conducted in low and middle income countries depicts a similar pattern of food consumption $[7,15]$. There exists definite evidence of bone depletion of the foetus during pregnancy, and thus adequate supplementation of calcium, phosphorus, and vitamin $\mathrm{D}$ during pregnancy is required [14]. Further research to analyse factors affecting lower intake of micronutrients and dietary patterns during pregnancy among women in Nepal is recommended.

Trimester-wise adequate nutrient intake has been recommended especially for energy and protein [19]. Thus, it is essential to observe trimester wise nutrient intake for positive pregnancy consequences. The nutrient intake per trimester for other nutrients apart from energy and protein has not been quantified by Indian Dietary Recommendations or any other guideline for pregnant women. The gradual increase in nutrient intake in pregnant women except for riboflavin, suggest pregnant women's willingness to eat more from the first to second and third trimester. The first trimester usually is accompanied by nausea and vomiting hence pregnant women usually skip the food [30]. The additional nutrient intake required per trimester has not been met as recommended by the IDR guidelines. This also implicates poor dietary patterns and this suggests less awareness among pregnant women regarding nutrient requirements during pregnancy as there is no evident practice of calorie/nutrient count.

The findings of this research suggest necessity to increase dietary intake such as whole grains, lentils, dairy products, meat products, eggs and fruits to supplement nutrients such as calcium, iron, vitamin C (ascorbic acid), vitamin B1 (thiamine), vitamin B2 (riboflavin) and vitamin B3 (niacin). Adequate food intake should definitely be preferred means for meeting nutrients requirements during pregnancy, however to meet nutrient needs during pregnancy is challenging with mere consumption of an adequate diet [31]. WHO recommendations for antenatal care for positive pregnancy outcomes recommends daily oral supplementation of iron and folic acid and context-specific recommendation for vitamin A and calcium supplementation for pregnant women to reduce the pregnancy related risks [10]. This highlights the importance of supplementation during pregnancy for positive pregnancy outcome.

For a detailed comparative study of other important nutrients, comprehensive research and inclusion of information regarding other nutrients is required and recommended in the Nepalese Food Composition Table of Nepal. The study was limited to respondents visiting the out-patient department ward of TUTH in Kathmandu district for ANC service and thus could not be generalized across Nepal. However, the results of this research are valuable to design nutrition related health programs and also for research related to dietary pattern and nutrient intake during pregnancy as this paper presents and discusses important information related to dietary and nutrient intake pattern of pregnant women. Although a 24 hour dietary recall method and questionnaire has been used and is sufficient to estimate average nutrient intake, there can be variation in day to day food consumption with a single 24 hour recall. Similar to other research conducted on nutrient intake, this research may also lead to some inaccurate results due to recall bias, ran- dom error due to variation and inaccurate portion estimation even though appropriate measures have been taken to reduce them. The comparison regarding intake of carbohydrates, carotene, phosphorous, fibre and minerals couldn't be made as the Nepalese Food Composition Table 2017 provided no such recommended intake values for these nutrient groups. Trimester-wise comparison with RNI cannot be made as Nepalese Food Composition Table 2017 does not provide any trimester wise recommended intakes.

\section{Conclusion}

Although nutrient intake for fat met RNI, crucial nutrients such as protein, calcium, iron, ascorbic acid, thiamine, riboflavin, niacin and energy intakes were below RNI. Trimester-wise intake though increased over the period of trimester, was not sufficient, particularly for energy and protein. The intake of cereals, followed by pulses, legumes, vegetables, fruits, milk, meat or fish was found to be high among pregnant women. Based on the research, balanced intake of nutritious food was not found and that may increase the risk of malnutrition which might affect the health of both women and foetus. Thus, this research suggests the need for a balanced nutritional diet among pregnant women. The need for supplementation of micronutrients is required to fulfil nutrient requirement until and unless the practice of balanced nutritional diet among pregnant women is established.

\section{Declaration}

The Authors declare no conflicting interests and confirms no involvement in funding that might have influenced the outcome of this research.

\section{References}

1. Daba G, Beyene F, Fekadu H, Garoma W. Assessment of knowledge of pregnant mothers on maternal nutrition and associated factors in Guto Gida Woreda, East Wollega Zone, Ethiopia. Journal of Nutrition \& Food Sciences. 2013;3(6):1.

2. Marangoni F, Cetin I, Verduci E, Canzone G, Giovannini M, Scollo P, Corsello G, Poli A. Maternal diet and nutrient requirements in pregnancy and breastfeeding. An Italian consensus document. Nutrients. 2016 Oct;8(10):629.

3. Organization WH. Essential nutrition actions: improving maternal, newborn, infant and young child health and nutrition. 2013.

4. Beckhaus AA, Garcia-Marcos L, Forno E, Pacheco-Gonzalez RM, Celedón JC, Castro-Rodriguez JA. Maternal nutrition during pregnancy and risk of asthma, wheeze, and atopic diseases during childhood: a systematic review and meta-analysis. Allergy. 2015;70(12):1588-604.

5. Keenan K, Bartlett TQ, Nijland M, Rodriguez JS, Nathanielsz PW, Zürcher NR. Poor nutrition during pregnancy and lactation negatively affects neurodevelopment of the offspring: evidence from a translational primate model. The American journal of clinical nutrition. 2013;98(2):396-402.

6. Alonso EB. The impact of culture, religion and traditional knowledge on food and nutrition security in developing countries. 2015.

7. Lee SE, Talegawkar SA, Merialdi M, Caulfield LE. Dietary intakes of women during pregnancy in low-and middle-income countries. Public health nutrition. 2013;16(8):1340-53.

8. Torheim LE, Ferguson EL, Penrose K, Arimond M. Women in resource-poor settings are at risk of inadequate intakes of multiple micronutrients. The Journal of nutrition. 2010;140(11):2051S-8S.

9. Kimmel MC, Ferguson EH, Zerwas S, Bulik CM, Meltzer-Brody S. Obstetric and gynecologic problems associated with eating disorders. International journal of eat- 
ing disorders. 2016 Mar;49(3):260-75

10. Mulligan ML, Felton SK, Riek AE, Bernal-Mizrachi C. Implications of vitamin $D$ deficiency in pregnancy and lactation. American journal of obstetrics and gynecology. 2010 May 1;202(5):429-e1.

11. Organization WH. WHO recommendation on antenatal care for positive pregnancy experience. Geneva: WHO; 2016 [cited 2018 Mar 25].

12. Status IoMSoN, Pregnancy WGd, Intake IoMSoD, Pregnancy NSd. Nutrition during pregnancy: part I, weight gain: part II, nutrient supplements: Natl Academy Pr; 1990.

13. Ministry of Health - MOH/Nepal, New ERA/Nepal, ICF. Nepal Demographic and Health Survey 2016. Kathmandu, Nepal: MOH/Nepal, New ERA, and ICF; 2017.

14. Nicholas H, Kuhn EM. The role of calcium, phosphorus and vitamin D in pregnancy. The Journal of clinical investigation. 1932;11(6):1313-9.

15. Andersen LT, Thilsted SH, Nielsen BB, Rangasamy S. Food and nutrient intakes among pregnant women in rural Tamil Nadu, South India. Public health nutrition. 2003;6(2):131-7.

16. Sahu KK, Idris M, Agarwal M, Singh S, Manar M. Dietary intake of pregnant women and its effect on the birth weight of newborns in rural area of Uttar Pradesh, India. Asian Journal of Medical Sciences. 2015;6(1):67-70.

17. Bhandari S, Sayami JT, Thapa P, Sayami M, Kandel BP, Banjara MR. Dietary intake patterns and nutritional status of women of reproductive age in Nepal: findings from a health survey. Archives of public health. 2016;74(1):2.

18. Institute of Medicine (US). Food and Nutrition Board. Dietary reference intakes: a risk assessment model for establishing upper intake levels for nutrients. National Academies Press. 1998.

19. Manual A. Dietary guidelines for Indians. Nat Inst Nutrition, Second edition. Hyderabad, India. 2011:89-117.

20. Annual Report - Department of Health Services 2074/75 (2017/18). Department of Health Services: Government of Nepal, Ministry of Health and Population, Services DoH; 2017/18 2019.

21. T.U. Teaching Hospital iom.edu.np: Institute of Medicine; [Available from: iom. edu.np/?page_id=132.

22. Sah R, Subedi L, Shah U, Jha N, Pokharel P. Nutritional supplementation practices during pregnancy in Village Development Committees of Morang District, Nepal. Journal of College of Medical Sciences-Nepal. 2014;10(2):10-7.

23. Biro G, Hulshof KF, Ovesen L, Cruz JA. Selection of methodology to assess food intake. European journal of clinical nutrition. 2002 May;56(2):S25-32.

24. Gibson RS, Ferguson EL. An interactive 24-hour recall for assessing the adequacy of iron and zinc intakes in developing countries. Washington, DC: ILSI Press; 1999.

25. Association WM. World Medical Association Declaration of Helsinki. Ethical principles for medical research involving human subjects. Bulletin of the World Health Organization. 2001;79(4):373.

26. Department of Food Technology and Quality Control. Nepalese Food Composition Table 2017. 2017.

27. Acharya O, Zotor FB, Chaudhary P, Deepak K, Amuna P, Ellahi B. Maternal nutritional status, food intake and pregnancy weight gain in Nepal. Journal of Health Management. 2016;18(1):1-12.

28. Koletzko B, Lien E, Agostoni C, Böhles H, Campoy C, Cetin I, Decsi T, Dudenhausen JW, Dupont C, Forsyth S, Hoesli I. The roles of long-chain polyunsaturated fatty acids in pregnancy, lactation and infancy: review of current knowledge and consensus recommendations. Journal of perinatal medicine. 2008 Jan 1;36(1):5-14. 29. Kramer MS, Kakuma R. Energy and protein intake in pregnancy. Cochrane Database of systematic reviews. 2003(4).

30. Latva-Pukkila U, Isolauri E, Laitinen K. Dietary and clinical impacts of nausea and vomiting during pregnancy. Journal of Human Nutrition and Dietetics. 2010
Feb;23(1):69-77.

31. Gernand AD, Schulze KJ, Stewart CP, West Jr KP, Christian P. Micronutrient deficiencies in pregnancy worldwide: health effects and prevention. Nature Reviews Endocrinology. 2016;12(5):274. 